Editor's Note: These short, critical reviews of recent papers in the Journal, written exclusively by graduate students or postdoctoral fellows, are intended to summarize the important findings of the paper and provide additional insight and commentary. For more information on the format and purpose of the Journal Club, please see http://www.jneurosci.org/misc/ifa_features.shtml.

\title{
The Role of Rostral Prefrontal Cortex in Establishing Cognitive Sets: Preparation or Coordination?
}

\author{
Roland G. Benoit \\ Institute of Cognitive Neuroscience and Department of Psychology, University College London, London WC1N 3AR, United Kingdom \\ Review of Rowe et al. (http://www.jneurosci.org/cgi/content/full/27/48/13303)
}

The prefrontal cortex (PFC) is thought to support processes that facilitate behavioral adaptation to the changing environment. It has been proposed that neurons in this region modulate links between perceptual inputs and appropriate actions according to currently expected reward contingencies (Miller and Cohen, 2001). This top-down influence exerted by the PFC thus allows for different responses to the same stimulus by establishing contextdependent sets. These are neurocognitive states that enable adaptation to imminent demands by preparing appropriate mental processes or responses. Previous neuroimaging evidence links the rostral PFC with the selection of such sets (Sakai and Passingham, 2003), whereas evidence from patients with lesions to this area suggests that its involvement may be limited to situations in which an action plan is not cued by the environment (Burgess et al., 2000).

Recently, Rowe et al. (2007) scrutinized whether rostral PFC is essential for initiating context-dependent processes in caudal brain regions (including more posterior PFC areas). Patients with lesions to

Received Jan. 16, 2008; revised Feb. 13, 2008; accepted Feb. 18, 2008.

This work was supported by a University College London Graduate School Scholarship and the German Academic Exchange Service. I thank Prof. Paul Burgess, Dr. Sam Gilbert, and Dr. Nico Bunzeck for helpful comments on a previous draft of this manuscript.

Correspondence should be addressed to Roland G. Benoit, Institute of Cognitive Neuroscience, Alexandra House, 17 Queen Square, London WC1N 3AR, UK. E-mail: r.benoit@ucl.ac.uk.

DOI:10.1523/JNEUROSCI.0206-08.2008

Copyright $\odot$ 2008 Society for Neuroscience $\quad$ 0270-6474/08/283259-03\$15.00/0 left rostrolateral PFC [Rowe et al. (2007), their Fig. 2 (http://www.jneurosci.org/ cgi/content/full/27/48/13303/F2), Table 1 (http://www.jneurosci.org/cgi/content/ full/27/48/13303/T1)] and healthy controls engaged in a working memory task while being scanned by functional magnetic resonance imaging (fMRI) (Fig. 1). In this way, Rowe et al. (2007) pioneered the combined use of human lesion and neuroimaging methods in the investigation of the functions subserved by this brain region. Each trial of the working memory task started with a cue instructing which of two stimulus types (i.e., spatial positions or letters) was currently relevant. After a delay, a series of both stimulus types was presented, but participants were required to remember the sequence of the cued stimuli only.

The patients exhibited selective deficits on switch trials (when the relevant stimulus type had changed compared with the last trial). In contrast, they actually made fewer errors than control subjects on stay trials (when the relevant stimulus type was the same as on the previous trial) [Rowe et al. (2007), their Fig. 3C (http://www. jneurosci.org/cgi/content/full/27/48/ $13303 / \mathrm{F} 3)]$. This was suggested to reflect a tendency for the patients to perseverate on the previously performed cognitive set (see below).

Concerning the fMRI data, Rowe et al. (2007) observed stimulus-type-specific set activations in caudal PFC regions during the instruction delay period, i.e., before stimulus presentation [Rowe et al. (2007), their Fig. 4 (http://www. jneurosci.org/cgi/content/full/27/48/ 13303/F4)]. Preparing to remember a series of positions was associated with recruitment of the frontal eye fields among other regions, whereas more ventral regions, such as Broca's area, were implicated in preparation for a sequence of letters. The authors took these activation patterns to reflect the implementation of processes relevant for remembering the respective stimuli.

It was hypothesized that if rostral PFC initiates set selection, the integrity of this structure would be necessary for preparatory activation of the more caudal, stimulus-type-specific brain regions. However, this was not the case: during the instruction delay, activation of these regions was not consistently weaker for the patients than for the controls [Rowe et al. (2007), their Fig. 5 (http://www. jneurosci.org/cgi/content/full/27/48/ 13303/F5)], suggesting that rostral PFC is not required for implementation of stimulus-type-specific neural sets when the relevant behavior is cued by the environment. The results reported by Rowe et al. (2007), however, do not rule out an involvement of rostral PFC in set selection in ill-structured situations, that is, when actions are not guided by environmental cues (Burgess et al., 2000).

Despite observing no consistent differences in preparatory set activity between 
patients and controls, Rowe et al. (2007) reported remarkable group differences in the functional connectivity of the stimulus-type-specific regions. Connectivity varied as a function of the targeted stimulus type for both groups. At least on stay trials, the letter compared with the position instruction was associated with stronger connections between Broca's area and the fusiform word form area. Conversely, connections between the superior frontal sulcus and the parietal cortex were greater for the position than the letter condition [Rowe et al. (2007), their Fig. $6 B$ (http://www.jneurosci.org/ cgi/content/full/27/48/13303/F6)]. Intriguingly, the connectivity was generally diminished for lesion patients, and was particularly weak on stay trials [Rowe et al. (2007), their Fig. 6A (http://www. jneurosci.org/cgi/content/full/27/48/ 13303/F6)]. This contrasts with the performance of patients, who made fewer errors than control subjects on these trials despite exhibiting especially reduced "functional brain connectivity."

The authors explained the behavioral effect by hypothesizing the existence of a default strategy, in which the subject engages the same task as in the previous trial. Only actively establishing the new task would overcome this predisposition on switch trials. Thus, Rowe et al. (2007) concluded that patients strongly relied on this default strategy, which impaired their performance on switch trials but led to improvements on stay trials.

There may be an alternative explanation for the data that Rowe et al. (2007) did not consider. Because the relevant stimulus type changed pseudorandomly across trials, participants had little foreknowledge about the upcoming task. Studies of task switching suggest that alternative sets are kept in a comparable state of readiness when the task sequence is unpredictable. Monsell et al. (2003) argued that this is because fully discarding a currently irrelevant cognitive set would be costly, because it might have to be reconfigured on the next trial. This hypothesis may account for the patients' superior performance on stay trials. That is, if patients did not concurrently maintain the alternate set, their performance would not have been subject to interference from it. This would lead to more errors on switch trials, because the now-relevant task set would have to be completely reestablished. This interpretation suggests that reduced overall functional connectivity during stay trials might reflect reduced connectivity of regions subserving the al-

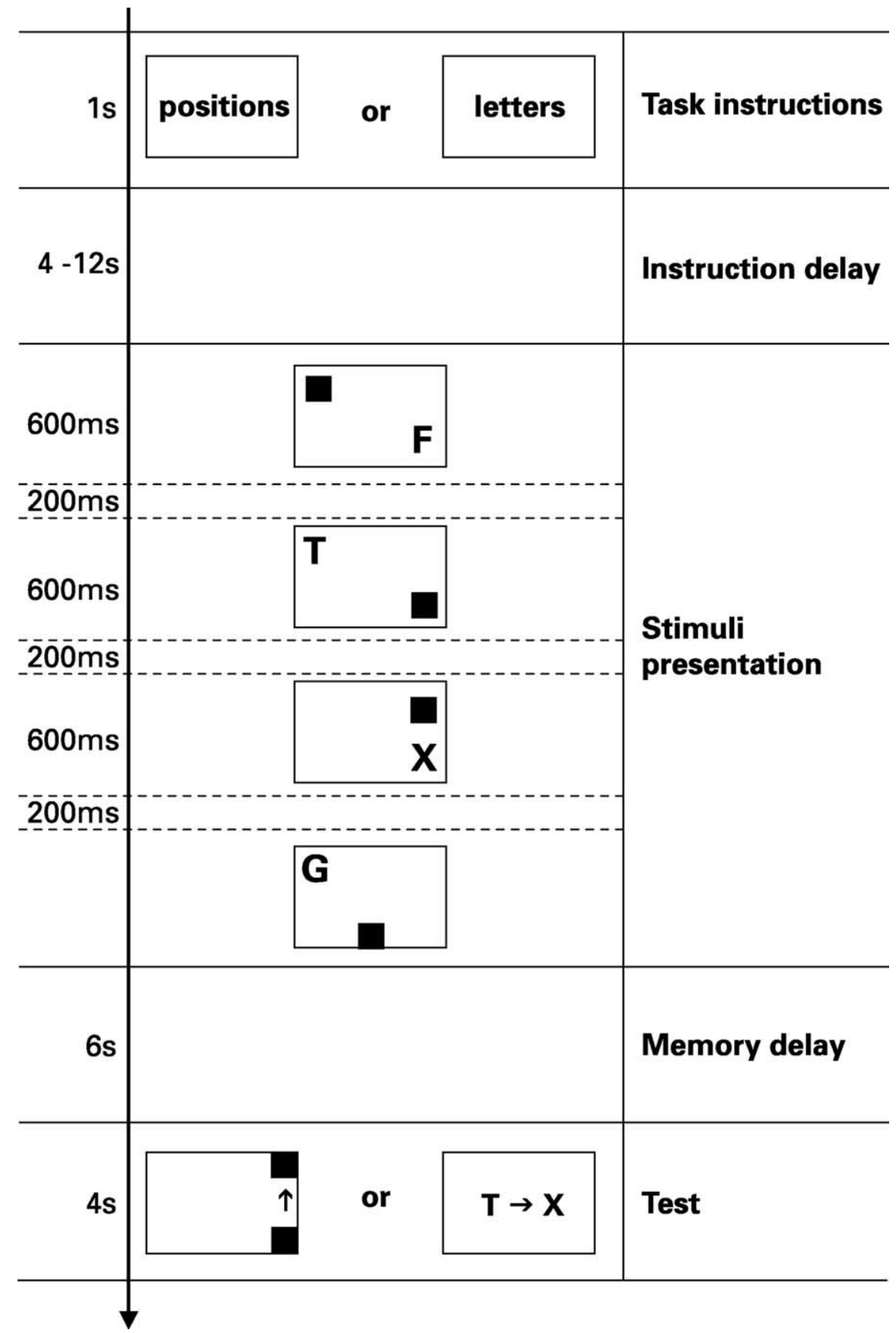

Figure 1. Illustration of the working memory paradigm. A task cue indicated the stimulus type that had to be remembered on a given trial (i.e., positions or letters), thus allowing for advance implementation of the currently relevant cognitive set. After an instruction delay, which lasted between 4 and $12 \mathrm{~s}$ (in steps of $2 \mathrm{~s}$ ), a series of four stimulus displays was presented. These comprised both spatial positions and letters, but only the cued stimuli had to be attended to. The appropriate sequence had to be maintained for a memory delay of $6 \mathrm{~s}$ and was tested by a single probe that required a judgment about the order of two of the stimuli (e.g., "Was the bottom right square immediately followed by the top right square?" or "Was the letter T immediately succeeded by the letter X?" "Yes" is the correct answer in both examples.).

ternate stimulus-type-specific set. Note, however, that the interaction between connection, stimulus type, switch-stay, and group was not significant $\left(F_{(1,21)}=\right.$ $1.0 ; p<0.33)$. Despite not being supported by this null result (which arguably might reflect a lack of statistical power), this alternative account is consistent with suggestions from other authors that pro- cesses supported by rostrolateral prefrontal cortex are involved in maintaining a cognitive set in a pending state during performance of another task (Simons et al., 2006; Koechlin and Hyafil, 2007).

Rowe et al. (2007) contribute valuable insights into the neural implementation of cognitive sets, and are the first to examine patients with rostral PFC lesions using 
fMRI. Because neuroimaging data per se is merely correlational, their results provide intriguing constraints for theorizing about the functions subserved by this region. Although left lateralized rostral PFC lesions do not necessarily affect the magnitude of set activation, they seem to generally diminish the functional connectivity among implicated cortical areas. However, as Rowe et al. (2007) pointed out, the absence of group differences in set activation may reflect their patient selection criteria. Because only patients with chronic unilateral lesions took part in this study, it is possible that functions normally subserved by the damaged site were performed by contralateral homologous regions (although these did not show compensatory overactivation). Moreover, because lesions were exclusively left later- alized, the authors conceded that lesions to the right hemisphere might have had a greater impact on stimulus-type-specific activation during the instruction delay. There is increasing evidence that rather than being a functionally homogenous region, distinct aspects of rostral PFC might subserve separable cognitive functions (Burgess et al., 2007). Future studies may address whether these subregions are also differentially involved in establishing cognitive sets.

\section{References}

Burgess PW, Veitch E, Costello A, Shallice T (2000) The cognitive and neuroanatomical correlates of multitasking. Neuropsychologia 38:848-863.

Burgess PW, Dumontheil I, Gilbert SJ (2007) The gateway hypothesis of rostral prefrontal cortex (area 10) function. Trends Cogn Sci 11:290-298.
Koechlin E, Hyafil A (2007) Anterior prefrontal function and the limits of human decisionmaking. Science 318:594-598.

Miller EK, Cohen JD (2001) An integrative theory of prefrontal cortex function. Annu Rev Neurosci 24:167-202.

Monsell S, Sumner P, Waters H (2003) Task-set reconfiguration with predictable and unpredictable task switches. Mem Cognit 31:327-342.

Rowe JB, Sakai K, Lund TE, Ramsøy T, Schramm Christensen M, Baare WFC, Paulson OB, Passingham RE (2007) Is the prefrontal cortex necessary for establishing cognitive sets? J Neurosci 27: 13303-13310.

Sakai K, Passingham RE (2003) Prefrontal interactions reflect future task operations. Nat Neurosci 6:75-81.

Simons JS, Schölvinck M, Gilbert SJ, Frith CD, Burgess PW (2006) Differential components of prospective memory? Evidence from fMRI. Neuropsychologia 44:1388-1397. 